16 | 2013

NOVECENTO... E DINTORNI

«On ne naît pas... on le devient »

\title{
La figura del femminiello/travestito nella cultura e nel teatro contemporaneo napoletano
}

The Femminiello/Cross-Dresser in the Culture and the Contemporary Theatre

of Naples

Mariano D'Amora

\section{OpenEdition}

\section{Journals}

Edizione digitale

URL: http://journals.openedition.org/cei/1198

DOI: 10.4000/cei. 1198

ISSN: 2260-779X

\section{Editore}

UGA Éditions/Université Grenoble Alpes

Edizione cartacea

Data di pubblicazione: 30 juin 2013

Paginazione: 201-212

ISBN: 978-2-84310-245-5

ISSN: 1770-9571

\section{Notizia bibliografica digitale}

Mariano D'Amora, «La figura del femminiello/travestito nella cultura e nel teatro contemporaneo

napoletano», Cahiers d'études italiennes [Online], 16 | 2013, online dal 15 décembre 2014, consultato il 27 mars 2021. URL: http://journals.openedition.org/cei/1198; DOI: https://doi.org/10.4000/cei.1198 


\title{
LA FIGURA DEL FEMMINIELLO/TRAVESTITO NELLA CULTURA E NEL TEATRO CONTEMPORANEO NAPOLETANO
}

\author{
Mariano D'Amora \\ Napoli
}

La cultura partenopea ha avuto, per vari decenni, quale suo esponente di rilievo il femminiello ${ }^{\text {I }}$. Espressione di un mix complesso di elementi socio-antropologici, questa figura era parte integrante del tessuto sociale dei quartieri popolari del centro storico di Napoli. Qui, egli godeva di una popolarità e benevolenza grazie anche alla sua partecipazione ad alcune manifestazioni folcloristiche e religiose. Nota è, infatti, la devozione dei femminielli per la Madonna di Montevergine ${ }^{2}$. Devozione che si manifestava nella ricorrenza della Candelora (il 2 febbraio) e nel pellegrinaggio dei devoti che dal centro di Napoli raggiungevano il Santuario di Montevergine, tra canti religiosi e balli tradizionali, accompagnati dal suono di nacchere e tammorre in un sincretismo religioso tra sacro e profano condiviso con tutta la comunità ${ }^{3}$. Questi balli hanno il nome di "Tammurriata», così descritta da Gorgoni e Rollin:

I. Un'esaustiva descrizione di come questa figura si presentava negli anni Ottanta viene fornita da Simonelli e Carrano: «I femminielli sono uomini che "vivono" e "sentono" da donna: abbigliati e truccati da donna. Spesso "prostitute" ma non necessariamente: ogni vicolo ha il suo femminiello accettato dalla comunità. Questi può vivere nella famiglia d'origine, attende alle occupazioni tradizionalmente riservate alle donne: cucinare, cucire, lavare la biancheria, fare le pulizie.» (Pino Simonelli e Giorgio Carrano, Le mariage des femminielli à Naples, in «Masques. Revue des Homosexualités», I8, 1983, pp. I05-I06.)

2. Il Santuario di questa Madonna è in provincia di Avellino sul massiccio montuoso del Partenio. L'agiografo Bargellini parlando della fondazione del Santuario di Montevergine da parte di san Guglielmo, riferisce che avvenne sul Partenio presso le rovine di un tempio dedicato alla dea pagana Cibele. I momenti fondanti del culto di Cibele erano: l'ascesa verso il monte sacro, l'adorazione di una pietra sacra e la presenza di fedeli maschi travestiti da donna che manifestavano una devozione frenetica ed estatica con canti e danze sfrenate al suono di tamburi e cimbali, cfr. Piero Bargellini, Mille santi del giorno, Firenze, Vallecchi, Massimo, 1977, p. I5.

3. Anche in tempi recenti la processione verso il Santuario ha conservato antiche peculiarità: «La prima sosta avviene in prossimità di una grotta dove si trova un rozzo sedile in pietra, antico ricordo della pietra sacra del culto di Cibele. Poi si riparte per raggiungere la scala santa, una scalinata di ventitré gradini che spesso si 
È un ballo, un canto, un suono, una delle maggiori espressioni musicali e sociali della tradizione folcloristica della Campania. Essa si è sempre realizzata, sin da tempi remoti, e continua a realizzarsi in tutta la sua solare estemporaneità, ad opera del popolo cafone, nelle campagne della provincia. Il fenomeno della tammurriata è legato soprattutto a momenti ritualizzati della collettività e con più precisione alla sacralità devozionale rivolta alle tante Madonne campane e a Sant'Anna. ${ }^{4}$

La partecipazione dei femminielli alle lodi per la Madonna nera, la "Mamma schiavona» (chiamata così perché il suo manto nero ricorda il colore della pelle degli schiavi), precede la costruzione del Santuario stesso poiché risalente al 1256 , anno in cui due omosessuali furono cacciati dalle mura cittadine per atti considerati osceni e portati sul monte Partenio affinché morissero di stenti e gelo in una giornata d'inverno. La leggenda narra che il miracolo si compì, e oltre al sole che squarciò le tenebre i due potettero anche accoppiarsi secondo le leggi di natura. Altro esempio paradigmatico di quanto profonde siano le radici di alcune pratiche appartenenti al mondo dei femminielli è costituito dalla cosiddetta "figliata d'e femminielli», un rituale derivante dall'antico rito della fecondità, praticato per secoli in Campania, riprova della convivenza a Napoli dell'aspetto teatrale con la dimensione sociale. La figliata si svolgeva alle pendici del Vesuvio, nella città di Torre del Greco e fu descritta accuratamente da Malaparte nel suo libro La pelle:

Era un uomo, senza dubbio, un giovane di non più di vent'anni. Si lamentava cantando a bocca aperta, e dondolava la testa qua e là sul guanciale, agitava fuor dei lenzuoli le braccia muscolose strette nelle maniche di una femminile camicia da notte, come se non potesse più sostenere il morso di qualche sua crudele doglia, e ogni tanto si toccava con ambe le mani, cantando: «ohi! ohi misera me!» il ventre stranamente gonfio, proprio il ventre di una donna incinta. [...] Pallido, gli occhi sbarrati, le due mani strette intorno alle tempie, il partoriente sbatteva il capo qua e là sul guanciale, gridando con voce acutissima. Una bava sanguigna gli schiumava intorno alle labbra, e grosse lacrime gli solcavano il bruno e maschio viso, imperlandogli i neri baffi. «Ciccillo! Ciccillo!» gridò la vecchia gettandosi sul letto e, ficcate le mani sotto le lenzuola, soffiando, facendo schioccar la lingua, sconciamente rumoreggiando con le labbra, stralunando gli occhi, e traendo su dal profondo del seno gorgoglianti sospiri, andava travagliando intorno a quel gonfio ventre, che ora si alzava, ora si abbassava, dondolando goffamente sotto la coperta di seta gialla. [...] A un tratto la vecchia si mise a tirare a sé con ambe le mani qualcosa fuor dal ventre di Ciccillo, e finalmente

percorrono in ginocchio intonando strofe, infine ci si presenta al cospetto della santa Madre.» (Paolo Valerio ed Eugenio Zito, Corpi sull'uscio, identità possibili. Il fenomeno dei femminielli a Napoli, Napoli, Filema, 20Io, p. 67.)

4. Patrizia Gorgoni e Gianni Rollin, Tammurriata, Napoli, Altra Stampa Edizioni, 1997, p. I4. 
con un grido di trionfo strappò, sollevò in alto, mostrò a tutti una specie di mostriciattolo di colore scuro, dal viso grinzoso sparso di macchie rosse. ${ }^{5}$

In origine, questa iniziazione a una femminilità sui generis avveniva durante i periodici festeggiamenti per l'avvenuta nascita del «maschiofemmina», dagli iniziati chiamata «Rebis», ossia res + bis, cosa doppia. Il rituale, descritto anche nella Napoli esoterica di Buonoconto ${ }^{6}$, richiedeva la presenza di un ermafrodito, l'unica creatura che contenesse i due elementi in cui è suddivisa tutta la natura. I greci, ritenevano divino l'ermafrodito, perché figlio della bellezza (Afrodite) e della forza (Ermes). Col tempo la purezza ideale dell'ermafrodito alchemico si è smarrita, sostituita dalla più materiale ambiguità del femminiello verso il quale, già dai primi del Novecento, una buona parte della letteratura piccolo-borghese partenopea non mancò di usare espressioni di profonda condanna morale:

Le nostre femminelle di giorno si occupano di faccende domestiche, e poi in ora stabilita si fanno alla finestra ed aspettano i loro... mariti. Parecchi di questi depravati, per rendersi più attraenti, si truccano gli occhi; altri si fanno, come le prostitute, tatuare sul viso qualche neo di bellezza e molti, mediante ovatta, cercano di rendersi più formose le parti posteriori e più sporgente il petto. Qualcuno si femminizza anche nel nome. ${ }^{7}$

Eppure l'amore non fu estraneo al loro vivere. Queste le parole scritte da Carluccia, femminella sedotta e poi abbandonata da Francesco T, ormai prossimo alle nozze:

Caro Ciccillo,

Io mi avveleno colle capuzzelle di fiammiferi, perché tu ammogliandoti, non potrai più abbracciare chi tanto ha sofferto per te arrivando a darti financo il suo onore. Del resto io ti perdono dell'offesa fattami perché sei cattivo come tutti gli altri uomini. In qualche momento della tua vita e delle tue gioie arricordati della tua affezionatissima amante.

Carluccia $^{8}$

I cambiamenti che dal dopoguerra in poi coinvolsero la cultura partenopea travolsero anche questa figura. La mutazione prese corpo gradualmente, cosicché il ruolo occupato tradizionalmente dal femminiello venne a modificarsi lasciando il posto al travestito. Ma, ancor prima dei

5. Curzio Malaparte, La pelle, Milano, Mondadori, I99I (prima edizione 1949), pp. I37-I38. La scena è ripresa anche dalla Cavani nel film omonimo tratto dal romanzo (I98I).

6. Cfr. Mario Buonoconto, Napoli esoterica. Un itinerario nei «misteri» napoletani, Roma, Newton Compton, 1996.

7. Abele De Blasio, Nel paese della camorra, Napoli, a spese dell'autore, I90I, p. 78.

8. Ibid., p. 8o. La lettera rappresenta un documento originale dell'epoca. 
mutamenti estetici ${ }^{9}$, è significativo comprendere le conseguenze dei cambiamenti sociali che investirono questa figura. Il prevalere dell'identità molteplice e cosmopolita della metropoli impose la perdita di centralità di questo personaggio all'interno del proprio contesto culturale, ossia: la solitudine del travestito in contrasto con la socialità del vicolo, che un tempo trovava aggregazione intorno al femminiello ${ }^{\text {Io }}$.

Quando, nei primi anni Ottanta, il teatro partenopeo decise di recuperare gli elementi fondanti della propria cultura popolare, ponendo al centro della sua riflessione le strade, le piazze, i colori, i suoni della Napoli contemporanea, non mancò di includere la figura del travestito, quale espressione di una napoletanità buia, a un passo dall'emarginazione. Tanto nelle Cinque rose di Jennifer (I98I) di Annibale Ruccello, quanto in Scannasurece (1982) di Enzo Moscato uno stato di lancinante solitudine permea la scrittura drammaturgica. Protagonista del primo dramma è Jennifer, un travestito. L'autore lo mostra nella sua casa situata in uno dei vari quartieri popolari sorti a Napoli tra il secondo dopoguerra e gli anni del boom economico. Luogo non definito, ma comunque limitrofo e, per giunta, abitato solo da travestiti. Mentre aspetta da mesi che torni il Franco conosciuto una sera di tanto tempo fa, Jennifer vive una vita domestica costantemente accompagnata da due elementi: la radio e il telefono. L'autore lascia la radio perennemente in funzione evidenziando in questo modo la condizione di solitudine del suo personaggio e creando, nel contempo, un dialogo tra musica e memoria, tra musica e vita. Si trasmettono i programmi di un'emittente locale, Radio Cuore Libero, interrotti solo da comunicati in cui si dà notizia di un maniaco che va uccidendo travestiti, sparando loro in bocca e lasciando sul cadavere cinque rose rosse:

VOCE DELLO SPEAKER: Si infittisce il mistero inerente agli omicidi nel nuovo quartiere dei travestiti. Stamani alle nove è stato scoperto un nuovo cadavere in un monolocale al terzo piano del numero sette di via del Cespuglio... ${ }^{\text {II }}$

Il telefono, a sua volta, squilla di continuo. Sembra che le linee telefoniche siano impazzite, tant'è che non si riesce mai a comunicare con la persona desiderata.

JENNIFER (con voce improvvisamente suadente): ... Pronto... (Eccitandosi) Franco sei tu! ... Ah... (Delusissimo si accascia su di uno sgabello) Scusate, tenevate la stessa voce... (Con dolcezza) A chi volete?... A Concetta?... E avete sbagliato numero, mi

9. Se nel primo caso gli indumenti indossati erano generalmente maschili, nel secondo si passa a indumenti evidentemente femminili.

IO. I quartieri di Napoli nei quali si registrava il più alto tasso di femminielli fra i residenti erano: Sanità, Pignasecca, Quartieri Spagnoli, Pallonetto, Borgo Sant'Antonio, Maddalena e Ferrovia.

II. Annibale Ruccello, Le cinque rose di Jennifer, Milano, Ubulibri, 2005, p. 25. 
dispiace... Questo è... (gentilissimo) il 42 6I 66... Concetta invece è il 25 5I 64... (Ridendo compiaciuto) No, grazie ma quello poi con tutte le interferenze che ci stanno dalla mattina alla sera me li sono imparati a memoria tutti i numeri del quartiere... ${ }^{\text {I2 }}$

Ma perché Ruccello sceglie un travestito quale protagonista del suo dramma? In merito all'uso di questo personaggio, l'autore dichiara di voler:

Analizzare in chiave antropologica l'universo dei travestiti, e più in generale quello della solitudine che a causa di modelli comportamentistici, culturalmente imposti, finisce per diventare una gabbia che impedisce di riscattarsi moralmente dalla solitudine. [...] L'essere travestito non è una scelta, bensì un'imposizione, è un ricalcare modelli già bruciati, a livello di conoscenza femminile. ${ }^{13}$

Vi è poi un'ulteriore funzione del travestito per il nostro drammaturgo, l'uso di questo personaggio come elemento di connessione con il teatro popolare:

Il segno infatti del travestirsi è alla base stessa del teatro popolare campano. Il travestimento più frequente è quello dell'uomo vestito da donna. C'è innanzitutto da considerare una componente sociologica che per secoli ha impedito alla donna di recitare e che ha fatto considerare l'attrice come una prostituta. Inoltre il travestimento corrisponde all'esigenza di far emergere nel momento del rituale tutto il represso quotidiano e quindi anche l'ermafroditismo e il desiderio di diventare donna almeno una volta l'anno. In più tale mascheramento corrisponde anche al tentativo di esorcizzare la parte femminile insita nella personalità maschile: essendo donna nel rito, in scena (cioè una volta l'anno) si è sicuri di essere uomini per i rimanenti 364 giorni. ${ }^{\text {I4 }}$

Ma si possono contare almeno altre due chiavi di lettura in merito a questo personaggio: una sociologica, l'altra culturale (sebbene le due siano strettamente collegate). Dal punto di vista sociologico il travestito innalza il rapporto simbiotico fra città e teatro al punto più alto. Egli, infatti, incarna la smarrita identità di Napoli negli anni Settanta-Ottanta. Simbolo di una città che, avendo perso se stessa (espressione con la quale si fa riferimento alla perdita di una propria identità culturale), si è di fatto auto-emarginata, abbandonandosi nell'attesa di un ipotetico roseo domani (come l'attesa da parte di Jennifer del suo amante Franco, figura forse mai esistita ma, probabilmente, nella sua idealità, frutto dell'immaginazione del personaggio) ${ }^{15}$. Inoltre nell'ambito di quel grande, ininterrotto, flusso di personaggi prodotti dalla drammaturgia napoletana nel

I2. Ibid., p. 24 .

13. Annibale Ruccello, Il teatro popolare in Campania, in Scritti inediti, a cura di Rita Picchi, Roma, Gremese, 2004, pp. 135-136.

I4. Ibid.

15. In riferimento al personaggio di Jennifer, Monaco scrive: «La parte negata della persona umana, quel doppio, che pur fondandosi nell'ordine sociale, non trova spazio nell'ordine sociale stesso» (Wanda Monaco, La contaminazione teatrale, Bologna, Pàtron, I98I, p. 225). 
secondo Novecento, Jennifer appare come naturale epigono di un travestito ancor precedente, Mariacallàs. Quando nel suo Persone naturali e strafottenti (1974) Patroni Griffi presenta in scena questo personaggio, egli apre una direzione inedita nel teatro partenopeo ${ }^{16}$. Come in Patroni Griffi, la condizione del travestito nel suo non essere sessualmente definito è metafora di una condizione esistenziale anch'essa in divenire. È pur vero, però, che Mariacallàs, trincerata dietro una maschera d'arroganza e strafottenza, riesce a far passare in secondo piano questa sua condizione: "MARIACALLAS: [...] Io proclamo. Sono una trombetta che strepita per le strade: fuori, fuori dalle case, vigliacchi, a raccolta! Sbalordimento e scandalo.» ${ }^{17}$ Diversamente, in Jennifer tale processo sembra ormai essere giunto a compimento, ogni barlume di esibito eroismo è decaduto. Lo stesso autore, sottolineando le intenzioni alla base del suo scritto, dichiara di voler: «[...] rappresentare l'impossibilità, per la solitudine, di rappresentarsi oggi come evento eroico. ${ }^{18}$ Jennifer non solo appare priva di una vita sociale e affettiva, ma le uniche relazioni con il mondo esterno sono rappresentate da una serie di grottesche telefonate e dalle visite di Anna (anch'egli un travestito). Non è più d'amore che si muore, ma di solitudine $^{19}$. Dal punto di vista culturale il travestito è espressione della doppia realtà della cultura napoletana contemporanea: da un lato quella ufficiale (o dello stereotipo potremmo dire) per quanti la percepiscono dall'esterno, dall'altro quella reale, per quanti la vivono dall'interno. Il duplice comportamento di Jennifer ne è la conferma. Di notte le sue inquietudini le offuscano la mente, di giorno celebra una personale epifania della rimozione vivendo come vorrebbe essere, uguale agli altri, con i luoghi comuni e le leziosità di qualunque altra donna di casa. Questo desiderio di fuga dalla realtà passa attraverso la finzione e attraverso il miraggio di un corpo altro da sé. Finzione che acquista ulteriore evidenza durante i suoi incontri con Anna. La condizione marcatamente surreale dei due personaggi che dialogano come fossero signore piccolo-borghesi alle prese con i relativi problemi familiari rende ancor più manifesto lo stato di isolamento e di incomunicabilità:

I6. Lo stesso Ruccello conferma la sua familiarità con l'opera di Patroni Griffi: «[...] non mi identifico con una drammaturgia nazionale. L'unica che esista, in questo momento, è napoletana. Qui c'è una tradizione, qui ci sono ben due padri spirituali tra i quali scegliere, Viviani ed Eduardo, c'è un suo sviluppo successivo con Patroni Griffi [...].» (Titti Marrone, Dalle «Rose» al «Weekend». Ė il momento di Ruccello, in "Il Mattino», 28 gennaio I986.)

17. Giuseppe Patroni Griff, Persone naturali e strafottenti, in Tutto il teatro, Milano, Mondadori, 1999, p. 39 I.

I8. Rossella Santilli, Jennifer o dell'ossessione, in «Napoli Oggi», I5 aprile I98I.

19. Il riferimento è ancora a Patroni Griffi e alla sua prima commedia D'amore si muore (1958). 
JENNIFER: ... Eh... Siete sposata?

ANNA: (visibilmente sorpreso) No!!... (Dubitativo) Signora... (Accettando il gioco) $\mathrm{O}$ almeno non ancora... E voi?

JENNIFER: (spedito) Eh!... la storia mia è complicata! Nu romanzo va’!... Sono stata sposata ben due volte... E mo' forse... mi sposo per la terza volta... (Molto «tè alle cinque») Eh!... Non ho avuto fortuna con i miei precedenti matrimoni...

ANNA: E... avete bambini?

JENNIFER: (prendendo il bricco del caffe) Sì... Due! ... Un maschietto e una femminuccia... il primo sta col padre... La seconda invece l'ho messa in collegio... Sta dalle Dorotee... A Roma... Sapete un bel collegio...

ANNA: («meravigliato»)... E come mai non l'avete tenuta con voi?! ... Quella poteva essere una compagnia...

JENNIFER: (mamma moderata) No Signora... Per carità! Io, i legami non mi piacciono... A me mi piace di essere libera... Ogni anno ci vediamo e passiamo insieme un mese di villeggiatura a Forte dei Marmi... (Epico)... Sapete... Dove va Mina!... (Abituale)... Io la incontro sempre... Ogni anno... Prima veniva con Paciuchino... Ma da quando è cresciuto, il ragazzo vuole la sua indipendenza e logicamente. Lei... ogni tanto... si lamenta che si sente sola, si sente sola... Ma io ce lo dico: «Mina sora mia, non ti attaccare troppo ai figli che dopo è peggio!» Del resto, come si dice... Devono fare la loro strada... ${ }^{20}$

Jennifer può essere considerata una figura 'deportata' dal punto di vista meramente urbanistico, ma socialmente è espressione piena e centrale di una cultura non più cultura, bombardata da una modernità intenzionalmente aggressiva, fino a smarrirsi in essa. E quanto il testo, o meglio il suo protagonista, sia per l'autore metafora espressiva dei mutamenti sociali che stavano avvenendo in Campania in quegli anni, lo dimostra la seconda versione del dramma. Negli anni immediatamente successivi al terremoto del novembre 1980, si spendono a Napoli cinquantamila miliardi di lire, dando vita a non meno di ventimila nuove abitazioni, (Pianura e le 'vele' di Scampia sono di questi anni). È interessante notare che, mentre nella prima versione del dicembre 1980 , Jennifer abita in una casa dei quartieri popolari e limitrofi (tipo Soccavo, o il rione Traiano), in un ambiente dai tratti marcatamente kitsch, ma pur sempre modesto, nelle versioni allestite in seguito (vedi quella del 1986) il personaggio si è trasferito in

20. Annibale Ruccello, Le cinque rose di Jennifer, cit., p. 3I. In realtà Mina è presente nel testo anche, e soprattutto come interprete musicale. Nella scaletta musicale indicata dall'autore compaiono cinque suoi brani: Quattrore e' tiempo, Grande grande grande, Ancora ancora ancora, Vorrei che fosse amore, Bugiardo e incosciente. 
una zona nuova della città ${ }^{21}$. La sua casa appare arredata con veneziane, adornata con lacche nere e uccelli d'oro, asettica nei funzionali cassetti che rientrano nelle pareti come la toilette e del secchio per l'immondizia. Anche i costumi sono segno dei mutamenti avvenuti. Jennifer ora indossa una vestaglia di raso bianco, un turbante, un abito di lamé, e per il suo lavoro di prostituta usa il vestito e la parrucca di China Blue, protagonista dell'omonimo film di Ken Russell ${ }^{22}$. La poetica alla base delle Cinque rose di Jennifer trova riscontro anche nella genesi di Scannasurece («ammazzatopi») di Enzo Moscato. Nel narrare la storia di un travestito confinato nel suo plumbeo monolocale-seminterrato nei Quartieri Spagnoli della città, l'autore presenta questo personaggio quale avanguardia paradossale e contraddittoria di un nuovo modo di concepire o di abolire i ruoli assegnati all'uomo e alla donna. Il personaggio diviene colui che si trova a sopportare stati di solitudine lancinanti, vittima di una società che lo isola, obbligandolo a vivere in quel modo, poiché ha paura di riconoscersi in lui. Come già Ruccello, anche Moscato utilizza questo personaggio in chiave sociologica, quale tramite per mettere in luce le ferite, le fratture provocate negli animi dei napoletani dalla storia e soprattutto dalla nuova e più grande insidia: la tentazione capitalistica verso l'omologazione. Nel mirino c'è quella Napoli da cartolina verso cui mostra una profonda insofferenza:

Posta com'è già sulla soglia di un vago — prossimo o già avvenuto, non si sa... sinistrico sfaldamento geocivile [...]; di un rovinar-decrepitar inarrestabile del Tutto, e, nel Tutto, in special modo, l'incancrenirsi (finalmente!) di quel suo stereotopico, folclorico, mandolinico, cartolinico "essere cantabile», che l'ha sempre, sciaguratamente, resa nota al mondo intero. ${ }^{23}$

Anche in questo caso viene offerta l'immagine di una città-cultura buia, suicida. In memoria di quella stessa solitudine che marchia la vita in scena di Jennifer, unici referenti, mal tollerati, di questo secondo travestito (cui l'autore non dà un nome) sono topi e studenti (quest'ultimi non appa-

2I. Il critico De Stefano ipotizza che l’abitazione post-terremoto di Jennifer sia da collocarsi a: «Scampia, alcuni insediamenti di edilizia popolare di Secondigliano, la periferia di San Giovanni, o quella di Ponticelli, la lista purtroppo è lunga" (Stefano De Stefano, Le cinque rose di Jennifer, in Annibale Ruccello e il teatro del secondo novecento, a cura di Pasquale Sabbatino, Napoli, Edizione Scientifiche, 2009, p. I20).

22. Nel programma di sala del 1986 Ruccello conferma i riferimenti cinematografici e sociali del testo: «L'attuale spettacolo vuole mettere in evidenza innanzitutto la doppia tessitura che hanno sempre i miei testi. Da un lato, appunto, la storia, una storia banale, in questo caso la giornata tipo di una persona in casa, le sue alienazioni, le sue manie, i suoi rituali privati, le sue vergogne e, soprattutto, il suo sentimento. Dall'altro il gioco delle citazioni, dei riferimenti, dei riporti da un fantastico principalmente filmico nella consapevolezza che oggi non è più possibile raccontare una storia se non soltanto per ammiccamenti, virgolettandola quasi, rendendola anch'essa null'altro che un repertorio di un immaginario il più possibilmente collettivo..."

23. Enzo Moscato, Introduzione, in Orfani Veleni, Milano, Ubulibri, 2007, p. 7. 
iono mai) ${ }^{24}$. I primi risultano ormai padroni della città, gli altri usano impropriamente la camera da letto del personaggio ${ }^{25}$. Nel corso di una giornata (da notare che entrambi i testi rispettano le tre unità aristoteliche di tempo, spazio e azione) quest'io narrante partecipa lo spettatore delle uniche tre peculiarità di Napoli sopravvissute: «[...] gli ipogei, 'a memoria e 'a magia... ben sapendo ca sule loro ce putevano salva'... ca sule loro putevano evita' le tarme, 'a naftalina o, peggio, di cadere seppelliti in qualche libbre, alla guisa di mummie alisandrine... ${ }^{26}$ Giunto al termine del suo personale delirio il personaggio si lascia andare a un'ira purificatrice distruggendo il monolocale ${ }^{27}$, prima di lanciare un'ultima lancinante domanda/invocazione a quel Dio così ostinatamente insensibile verso questa terra: «Quando scoppia 'stu 'rilorgio? Quando? Pure 'o cielo allora, è 'nu bancone? E Dio cu' nuje è sulo 'nu struzzino?»" ${ }^{28}$ Il personaggio, dopo aver indossato un kimono giapponese, si suicida dando fuoco all'ambiente in cui vive:

va in un angolo, afferra una lattina colma di benzina. Comincia, quasi religiosamente in modo ieratico, a cospargere l'intero spazio di benzina. [...] apre la scatola di fiammiferi, ne tira fuori uno, lo tiene un attimo sospeso [...] lo accende e con un ultimo, largo gesto del braccio, lo getta... ${ }^{29}$

Ma la morte, per mano propria, avviene anche nel finale di Jennifer:

Jennifer $[\ldots]$ prende a sbattere il ricevitore. Prima piano, poi sempre più forte. Almeno è un rumore, un suono. Anzi, proviamo con lo sgabello. Proviamo. Sempre più forte. Sempre. Per terra. [...]. Percuotiamo la radio [...]. Jennifer crolla per terra, stracciandosi i vestiti, percuotendosi urlando "Mamma... Mamma». [...] La pistola. La punta. La bocca. Lavvicina. Cazzo sembra di fare un pompino. Buio. Torna la

24. Gli scambi fra Ruccello e Moscato su questo testo sono confermati da Gliozzi: «Mentre lui provava Scannasurice, nella stanza accanto Annibale lavorava a Le cinque rose di Jennifer. Hanno giocato spesso, in quel periodo, a scambiarsi la regia, contaminando l'uno i testi e le invenzioni sceniche dell'altro.» (Melanie Gliozzi, Nota biografica-artistica di Enzo Moscato, in Comicità negli anni settanta. Percorsi eccentrici di una metamorfosi fra teatro e media, a cura di Eva Marinai, Sara Poeta e Igor Vazzaz, Pisa, ETS, 2005, p. 97-I06; 99.) Non a caso, quando nel 1984 Moscato torna a lavorare su questo testo, allestendone la seconda versione, con diverso finale, a Torre del Greco nel Teatro nel Garage nell'aprile di quell'anno, la regia è curata da Annibale Ruccello. Enzo Moscato, Scannasurice, in Orfani Veleni, cit., pp. 37-39. Un'ulteriore elaborazione del testo avviene nel 1989 dando vita a Scanna-Play-Surice.

25. Riferimento a Persone naturali e strafottenti di Giuseppe Patroni Griffi, in Tutto il teatro, con una nota introduttiva di Paolo Bosisio, Milano, Mondadori, I999.

26. Enzo Moscato, Scannasurice, cit., p. I3.

27. Così in Moscato: «Va al tavolo rovescia tutto, con disperazione. Afferra i sacchetti della spazzatura e ne rovescia il contenuto per terra. Rompe bottiglie, abiti, oggetti di vetro, tutto quello che gli capita a tiro, un una sorta di furore, al tempo stesso angosciante e liberatorio, da invasato" (ibid., p. 34).

28. "Quando scoppia quest'orologio? Quando? Allora anche il cielo è un bancone? E Dio è solo uno strozzino con noi?» (Ibid., p. 36)

29. Ibid. 
luce. La luce dell'abat jour. Lui è lì. Riverso per terra. La casa in disordine, le solite cinque rose sul corpo. La pistola. Indifferente, squilla il telefono. ${ }^{30}$

Stigmatizzati come figure negative, entrambi i personaggi scelgono di togliersi la vita. Ogni forma di ribellione risulta vana, è possibile solo un'implosione violenta. Nella morte, essi trovano l'unico epilogo possibile, laddove il vissuto descritto dagli autori si pone quale negazione assoluta di ogni speranza verso il futuro. Tornano alla mente le parole di Pasolini sui napoletani, pronunciate pochi anni prima:

Io so questo: che i napoletani sono oggi una grande tribù che anziché vivere nel deserto o nella savana, come i Tuareg, vive nel ventre di una grande città di mare. Questa tribù ha deciso di estinguersi rifiutando il nuovo potere, ossia quella che chiamiamo la storia, o altrimenti la modernità. [...] Questo rifiuto è sacrosanto. ${ }^{31}$

Ebbene, è proprio quel «rifiuto» che i due drammaturghi vanno ad analizzare chiedendosi se sia vero che i loro conterranei hanno "deciso di estinguersi» o se, al contrario, hanno accettato le storture della modernità. L'isolamento in cui sono confinati i due personaggi diventa metafora espressiva dei dogmi imposti dalla cultura di massa che, sentendo minacciata la propria «sicurezza ontologica ${ }^{32}$, non lascia spazio a individui non pienamente rispondenti all'imperante conformismo piccolo-borghese. Essi sono quel confine in negativo oltre il quale non è bene spingersi. Se il femminiello era parte integrante della cultura del vicolo, quindi espressione degli aneliti culturali più intimi della città, la morte del suo epigono contemporaneo, ormai trattato come soggetto "deviante» ${ }^{33}$, porta con sé la fine di quella parte di cultura partenopea, avviatasi anch'essa verso l'omologazione ${ }^{34}$.

30. Annibale Ruccello, Le cinque rose di Jennifer, cit., pp. 42-43.

31. Dichiarazione di Pier Paolo Pasolini rilasciata ad Antonio Ghirelli nel 1971, in seguito pubblicata in Antonio Ghirelli, La napoletanità, Napoli, Società Editrice Napoletana, 1976, pp. I5-I6. Ora in Pier Paolo Pasolini, La napoletanità, in Saggi sulla politica e sulla società, edizione diretta da Walter Siti e Silvia De Laude, vol. I, Milano, Mondadori, I999, p. 230.

32. Cfr. Anthony Giddens, Identità e società moderna, Napoli, Ipermedium, I999.

33. La definizione di «deviante» da parte di Ambrogio Santambrogio bene esprime la percezione di questa figura da parte della cultura di massa: «Il deviante — se pur non diventa un vero e proprio capro espiatorio costituisce allora una fonte di sicurezza per l'identità collettiva: quest'ultima, piuttosto che fondarsi su sicurezze in positivo, ricorre a sicurezze in negativo.» (Ambrogio Santambrogio, Introduzione alla sociologia delle diversità, Roma, Carocci, 2003, pp. 93-94.)

34. La figura del femminiello e/o del travestito ricorre sovente nella drammaturgia e nella narrativa napoletana dal secondo Novecento in poi. Michele Serio ne parla nel suo romanzo Nero metropolitano (Milano, Dalai, 1996) così come Andrej Longo in Adelante (Milano, Rizzoli, 2003); Roberto De Simone nel celebre spettacolo teatrale Gatta Cenerentola inserisce il personaggio del femminiello quale protagonista di due scene chiave dell'opera: il rosario dei femminielli e il suicidio del femminiello. Oltre che in Persone naturali e strafottenti, Giuseppe Patroni Griffi sceglie un travestito (Rosalinda Sprint) quale protagonista del suo romanzo Scende giù per Toledo (1975). Nel $201 \mathrm{I}$ a cura del sottoscritto vengono pubblicati per l'editore Bulzoni di Roma alcuni 


\section{Bibliografia}

Bargellini Piero, Mille santi del giorno, Firenze, Vallecchi, Massimo, 1977.

Baker Roger, Drag. A History of Female Impersonation on Stage, London, Triton, I968.

Buonoconto Mario, Napoli esoterica. Un itinerario nei «misteri» napoletani, Roma, Newton Compton, 1996.

Comicità negli anni settanta. Percorsi eccentrici di una metamorfosi fra teatro e media, a cura di Eva Marinai, Sara Poeta e Igor Vazzaz, Pisa, ETS, 2005 .

D’Amora Mariano, Male Transvestitism: Bridging Present and Past in Contemporary Drama, in Differences on stage, a cura di Alessandra De Martino, Paolo Puppa e Paola Toninato, Cambridge, Cambridge Scholars Publishing, in corso di stampa.

De Blasio Abele, Nel paese della camorra, Napoli, a spese dell'autore, I9OI.

De Stefano Stefano, Le cinque rose di Jennifer, in Annibale Ruccello e il teatro del secondo novecento, a cura di Pasquale Sabbatino, Napoli, Edizioni Scientifiche Italiane, 2009, pp. II7-I24.

De Simone Roberto, Il travestimento da donna e altre maschere, in Annabella Rossi e Roberto De Simone, Carnevale si chiamava Vincenzo, Roma, De Luca Editore, 1977, pp. 209-222.

Gargiulo Giuliana, Napoli nuda vestita e travestita, Napoli, Colonnese, 1999.

Giddens Anthony, Identità e società moderna, Napoli, Ipermedium, 1999. Ghirelli Antonio, La napoletanità, Napoli, Società Editrice Napoletana, 1976.

Gorgoni Patrizia e Rollin Gianni, Tammurriata, Napoli, Altra Stampa Edizioni, 1997.

Heath Kirk, Uomo Donna. Il mondo dei travestiti in teatro, Como, Lyra Libri, 1987.

Longo Andrej, Adelante, Milano, Rizzoli, 2003.

testi teatrali di Fortunato Calvino. Fra questi c'è Vicolo Sirene. Personaggi principali sono dei travestiti, anche in questo caso vittime di emarginazione culturale e fisica. Segnalo inoltre, quale preziosa fonte d'informazioni per il vissuto dei travestiti a Napoli oggi, il testo di Giuliana Gargiulo, Napoli nuda vestita e travestita, Napoli, Colonnese, 1999. Un'approfondita analisi della figura del travestito nei carnevali campani è sviluppata da De Simone in Il travestimento da donna e altre maschere, in Annabella Rossi e Roberto De Simone, Carnevale si chiamava Vincenzo, Roma, De Luca Editore, 1977, pp. 209-222. 
Malaparte Curzio, La pelle, Milano, Mondadori, I99i (prima edizione I949).

Marrone Titti, Dalle «Rose» al «Weekend». Ė il momento di Ruccello, in «Il Mattino», 28 gennaio 1986.

Monaco Wanda, La contaminazione teatrale, Bologna, Pàtron, 198I.

Moscato Enzo, Orfani Veleni, Milano, Ubulibri, 2007.

Pasolini Pier Paolo, La napoletanità, in Saggi sulla politica e sulla società, edizione diretta da Walter Siti e Silvia De Laude, vol. I, Milano, Mondadori, 1999, pp. 230-23I.

Ruccello Annibale, Le cinque rose di Jennifer, Milano, Ubulibri, 2005.

-, Il teatro popolare in Campania, in Scritti inediti, a cura di Rita Picchi, Roma, Gremese, 2004.

Patroni Griffi Giuseppe, Persone naturali e strafottenti, in Tutto il teatro, Milano, Mondadori, I999.

Santambrogio Ambrogio, Introduzione alla sociologia delle diversità, Roma, Carocci, 2003.

SAntilli Rossella, Jennifer o dell'ossessione, in «Napoli Oggi», Is aprile I98I.

Serio Michele, Nero metropolitano, Milano, Dalai, 1996.

Simonelli Pino e Carrano Giorgio, Le mariage des femminielli à Naples, in «Masques. Revue des homosexualités», I8, 1983, pp. I05-II6.

Valerio Paolo e Zito Eugenio, Corpi sull'uscio, identità possibili. Il fenomeno dei femminielli a Napoli, Napoli, Filema, 2010. 